Service social

\title{
Histoires de familles et de réseaux - La sociabilité au Québec d'hier à demain, par Andrée Fortin avec la collaboration de Denys Delage, Jean-Didier Dufour et Lynda Fortin, Les Éditions coopératives Allbert Saint-Martin, Montréal, 1987, 225 pages.
}

\section{Jacques Roy}

Volume 37, numéro 3, 1988

Index analytique 1978-1988

URI : https://id.erudit.org/iderudit/706415ar

DOI : https://doi.org/10.7202/706415ar

Aller au sommaire du numéro

Éditeur(s)

École de service social de l'Université Laval

ISSN

1708-1734 (numérique)

Découvrir la revue

Citer ce compte rendu

Roy, J. (1988). Compte rendu de [Histoires de familles et de réseaux - La sociabilité au Québec d'hier à demain, par Andrée Fortin avec la collaboration de Denys Delage, Jean-Didier Dufour et Lynda Fortin, Les Éditions coopératives Albert Saint-Martin, Montréal, 1987, 225 pages.] Service social, 37(3), 463-467. https://doi.org/10.7202/706415ar d'utilisation que vous pouvez consulter en ligne. 


\section{RECENSIONS}

Histoires de familles et de réseaux - La sociabilité au Québec d'hier à demain, par Andrée FORTIN avec la collaboration de Denys DELAGE, Jean-Didier DUfOUR et Lynda FORTIN, Les Éditions coopératives Albert Saint-Martin, Montréal, 1987, 225 pages.

La famille. Autant de modèles, de façons de vivre entre enfants et adultes. C'est aussi le premier lieu de solidarité que l'on investit. C'est également une sorte de charnière entre la personne et la communauté locale, le quartier, le village.

De toutes parts, les politiques sociales (en particulier celles visant la désinstitutionnalisation) convient la famille à un rôle plus prépondérant à l'égard de ses membres les plus fragiles, les plus malades. Mais au détour des orientations et des plans projetés, sera-t-elle au rendez-vous souhaité ? Pourra-t-on compter sur la famille pour prendre le relais des responsabilités qui, jadis, appartenaient à l'État?

Sans répondre de façon décisive à ces questions (ce n'était pas son objet principal), la recherche réalisée par Andrée Fortin, sociologue, apporte néanmoins un éclairage fort pertinent sur la nature et la sociabilité des familles et des réseaux naturels, d'hier à demain. Tel un carrelage géographique où se déploie la vie d'une douzaine de quartiers de la région de Québec, la recherche nous reproduit, par quartier, l'image de 370 familles qui y évoluent. Son intention était double : décrire la sociabilité des familles dites " ordinaires", c'est-à-dire nucléaires à leur première union, et explorer les nouvelles formes de solidarité et de sociabilité, non essentiellement familiales, de la famille de demain (monoparentale et reconstituée, incluant les individus qui ont peu de frères et sœurs, ou qui en sont coupés géographiquement).

\section{Familles d'hier et d'aujourd'hui : un trait d'union dans le temps}

La Révolution tranquille a-t-elle provoqué une rupture définitive avec le modèle de la famille traditionnelle d'antan? Pas tout à fait si l'on s'en remet aux données de l'enquête. Un chapitre porte même sur le phénomène des "villages en ville" où des quartiers, tel Saint-Sauveur dans la basse-ville de Québec, ont conservé dans le temps (et exporté en milieu urbain) les mêmes traits de sociabilité familiale que l'on retrouvait jadis dans les campagnes. Dans ces quartiers (souvent populaires), la parenté fait partie du voisinage et la sociabilité traditionelle a survécu : il se forme des clans familiaux et la parenté est toujours plus importante que les amis ou voisins.

Cette analyse rejoint celle de Fernand Dumont qui soulignait dans un récent ouvrage que «La première génération urbaine n'a pas délaissé d'un 
coup les mentalités et les traditions des campagnes. Ces mentalités et ces traditions se sont continuées en ville; elles s'y sont réaménagées afin de mieux durer ${ }^{1}$ ».

Cependant, ces formes de solidarité familiale et sociale observées doivent composer avec une métamorphose singulière de la famille et des réseaux naturels. C'est ainsi que la recherche dirigée par madame Fortin identifie trois types de réseaux de sociabilité : " Il y a d'abord le réseau "traditionnel", qu'on retrouve surtout en milieu populaire, dont l'unité de base est la famille étendue, "le clan ». On peut parler en second lieu de l'univers des couples, où ceux-ci sont l'unité de base des relations sociales; ils préexistent aux familles en tant que telles et leur donnent du sens; ce modèle n'est pas nouveau, il a " toujours » existé dans les classes moyennes et aisées. Enfin, on peut parler de nouveaux réseaux quand l'unité de base est l'individu. On ne valorise plus les liens du sang en tant que tels, mais les rencontres qu'ils permettent de réaliser entre hommes et femmes d'âges différents; les adultes ne sont pas perçus comme des moitiés de couple (réel ou potentiel) ni les enfants uniquement comme " les enfants de... ". Ce dernier type de réseau se rencontre un peu partout, à des degrés divers, dans les interstices des modèles précédents (gens qui ont peu de famille ou qui en sont éloignés; familles monoparentales), chez ceux et celles qui ont effectué une rupture culturelle et/ou idéologique avec les valeurs traditionnelles ${ }^{2}$ ". Ajoutons à cela que ce dernier réseau constitue le groupe montant : les familles monoparentales et reconstituées sont en passe de former la majorité des familles au Québec comme ailleurs.

Malgré cette diversité de familles et de réseaux sociaux, malgré le fait que les années 1970 aient proclamé bien haut la mort de la famille, cette dernière serait encore bien vivante dans l'esprit des gens, elle n'aurait pas perdu son sens premier : "Les valeurs familiales sont encore très fortes même si elles se sont transformées, même si elles sont véhiculées par des familles nucléaires coupées de leur famille élargie, même si elles sont vécues dans des familles monoparentales ${ }^{3} »$.

D'ailleurs, en entrevue, Andrée Fortin nous met en garde concernant le hiatus entre discours entendu sur la famille et réalité : "Il ne faut pas confondre les discours et la pratique, entre autres les discours normatifs quand ils sont tenus soit par l'Église à une époque donnée ou par les féministes, plus récemment. II faut croire enfin que c'était une élite intellectuelle qui projetait sa vision du monde ou son mode de vie et qui $s^{\prime}$ imaginait que tout le monde pensait comme elle ». Un exemple : les femmes dans la société traditionnelle : "Si on peut parler de patriarcat officiel - et les dispositions légales et religieuses ne laissent pas de doute là-dessus - on remarque en pratique une famille plus égalitaire, une place plus grande laissée à la femme que le discours officiel ne le laissait croire ${ }^{4} »$.

Mais forcément, des traits se transforment dans le temps. Ainsi, le phénomène de cohabitation : "La cohabitation des générations dans le même logement est assez rare, et se trouve entravée non seulement par les changements de mentalités et un plus grand désir d'indépendance, mais aussi par l'exiguïté des logements ${ }^{5}$ ». 
Également, l'enquête révèle que les enfants, au-delà de la diversité des types de familles et des formes d'habitation, sont le ciment des relations sociales entre parents et voisins, le passeport premier de la sociabilité : on les retrouve partout dans le quartier et ils sont souvent le motif des premiers échanges entre voisins. "Les enfants cherchent une famille et ils forcent parfois les adultes à réagir ", d'ajouter l'auteure.

Enfin, la recherche met en relief le phénomène de retour au centre-ville, où se mélange aux populations locales traditionnelles ce qu'on a surnommé "la petite bourgeoisie décapante ${ }^{6}$ »

\section{Hommes et femmes : des sociabilités différentes}

Lorsqu'on parle de familles, on ne peut faire autrement que d'évoquer les rapports entre hommes et femmes comme le terrain privilégié des transformations familiales. Pour Maurice Champagne, seule une révolution des rapports hommes/femmes pourrait sauver la famille dans l'avenir. Dans la foulée d'une conférence portant sur le thème "familles et culture ", Champagne soulignait à grands traits que "Les femmes ne veulent plus être les seules responsables de la qualité de la vie familiale et de la prise en charge des enfants. On veut que les hommes assurent la paternité aussi intensément que les femmes ont assumé la maternité à travers l'histoire ${ }^{7}$ ".

Mais que révèle l'enquête sur l'implication des hommes au niveau de la famille et des réseaux sociaux? D'abord qu'ils (familles et réseaux) sont essentiellement une affaire de femmes ${ }^{8}$. Ce sont elles qui établissent les relations, c'est par elles que l'on " passe " pour contacter la parenté, établir des liens avec le voisinage...

Également, l'enquête a permis de dégager deux modèles de sociabilité selon le sexe (dont l'un, la sociabilité masculine, est demeuré largement un mystère dans la recherche, selon Andrée Fortin). Pour l'essentiel, on associe le réseau féminin davantage à la parenté alors que le réseau des hommes est principalement axé sur les activités professionnelles. C'est ainsi que les rencontres, les relations, originent de deux canaux différents.

L'étude a pointé du doigt un phénomène particulier : celui des amitiés. Les femmes ont presque toujours une amie, pas les hommes; tout au plus, ils ont des copains, des camarades : "L'amitié entre hommes est rare et semble avoir besoin de conditions exceptionnelles pour naître et s'épanouir ${ }^{9}$ ". Les hommes semblent peu engagés dans leurs relations; lorsqu'il y a amitié, c'est souvent entre frères qu'elle a le plus de chance de se concrétiser.

Les hommes gravitent donc autour de l'univers du travail et, selon Andrée Fortin, le partage social des tâches familiales et domestiques n'est pas encore réalité. Les changements avanceraient dans la bonne direction mais à pas de tortue.

Malgré tout, Andrée Fortin est optimiste pour I'avenir. Comme un signe de demain, elle lance l'exemple des hommes monoparentaux; " on voit peu $d$ 'hommes monoparentaux mais quand on en voit, ils nous tiennent les mêmes discours que les femmes monoparentales; ils "embarquent " de la même façon, ils ont les mêmes complaintes ". L'auteure fait remarquer que selon les 
statistiques publiques, le nombre d'hommes prenant la garde des enfants après une séparation progresse. Mais lentement.

\section{Les discours officiels ou l'utopie communautaire}

On pénètre ici dans le cénacle des discours gouvernementaux où la famille est promue aux fonctions sociales de l'État, tant en matière de santé que sur le plan social et communautaire. Et pour cause : on estime à $70 \%$ et $80 \%$ la contribution de la famille en matière de soutien à domicile ${ }^{10}$. Cette nouvelle sollicitation plus formelle, plus sentie, intervient au moment même où d'aucuns considèrent la fragilité des réseaux naturels actuellement; où la famille voit sa taille fondre; où l'on se dirige vers une société à enfants uniques si les tendances démographiques et sociales se maintiennent.

Ce nouveau contexte inquiète vivement Andrée Fortin. Et pour plusieurs raisons. D'abord, une question de nombre : il y aura par exemple moins d'enfants pour soutenir les personnes âgées. Un dossier réalisé dans le cadre des travaux de la Commission Rochon soulève la question tout en posant de sérieuses interrogations sur le soutien familial dans I'avenir : "Jusqu'en 1961, le Québec comptait 30 personnes de 65 à 79 ans pour chaque 100 adultes de 40 à 54 ans. Aujourd'hui, la proportion de personnes âgées est de 45 et elle passera à plus de 80 en 2031. Ces projections démographiques indiquent la diminution de la disponibilité et du soutien familial ${ }^{11}$ ".

Ce qui signifie " qu'un même individu recevra davantage de demandes et de pressions pour soutenir et aider les personnes âgées, compte tenu du fait que la disponibilité d'aide sera numériquement plus faible ${ }^{12} »$. Ce qui n'est pas sans inquiéter l'auteure puisque, selon elle, ce sont principalement les femmes qui seront mises à contribution: "ce sont les femmes qui, traditionnellement, ont toujours assuré la dimension relationnelle. L'aide à la famille, le bénévolat dans le secteur de la santé, c'est aujourd'hui encore largement l'affaire des femmes. C'est pourquoi les politiques de désinstitutionnalisation risquent de reposer encore sur elles dans l'avenir ».

Mais en raison du phénomène de la double tâche chez les femmes (emploi et travail domestique), un retour sans nuance des responsabilités de I'État vers elles pourrait provoquer un phénomène de burn-out des réseaux naturels : "Tout le monde parle des réseaux; personne n'a été voir dans quel état ils sont "d'ajouter l'auteure de la recherche qui convient de l'intérêt de trouver les meilleures formules d'arrimage entre l'État, la famille et les réseaux naturels, mais pas au détriment de ces deux derniers, au risque de les épuiser, voire de tuer ce qui pourrait rester de solidarité chez eux.

"On oublie pourquoi on avait voulu institutionnaliser les personnes âgées, les malades... Chose certaine, on ne peut pas dire maintenant : " reprenez vos vieux, vos malades, car ce ne serait pas les mêmes familles qui les reprendraient " de préciser Andrée Fortin en référant à la métamorphose des familles d'aujourd'hui.

Le débat est ouvert. II ne remet pas en cause le caractère souhaitable des politiques de désinstitutionnalisation. II concerne plutôt les modalités, entre autres le recours aux familles et aux réseaux naturels pour partager les responsabilités des politiques sociales et de santé. À cet effet, l'enquête sert 
donc une mise en garde : "Le soin des malades, des handicapés et des personnes âgées était autrefois une responsabilité familiale, puis le flambeau est passé à l'État; cela est devenu une responsabilité gouvernementale. II est impensable de repasser, tel quel, le flambeau aux familles et aux individus... Les réseaux "naturels " sont déjà essoufflés dans bien des $\operatorname{cas}^{13}$ ".

Centre de recherche sur les services communautaires,

Jacques Ror

Université Laval.

\section{Références}

${ }^{1}$ Extraits du volume Le sort de la culture, Éditions de l'Hexagone, publiés dans Le Devoir, 19 décembre 1987, p. D1.

${ }^{2}$ Andrée Fortin et al., Histoires de familles et de réseaux, p. 87-88.

3 Ibid., p. 217.

4 Ibid., p. 16.

5 Ibid., p. 195.

6 Ibid., p. 147.

${ }^{7}$ Propos recueillis dans Le Soleil du 5 décembre 1987, p. A-17, suite à une conférence donnée à Paris et intitulée " La famille et la culture nord-américaine sous le choc de la modernité ".

${ }^{8}$ Andrée Fortin et al., op. cit., p. 66 et 175.

9 Ibid., p. 177.

${ }^{10}$ Commission d'Enquête sur les Services de Santé et les Services Sociaux, Dossier "personnes âgées ", programme de consultation d'experts, août 1987, p. 27.

11 Ibid., p. 57-58.

12 Ibid., p. 26.

${ }^{13}$ Andrée Fortin et al., op. cit., p. 221.

Adolescents Parents Communication Couples - comment mieux comprendre..., par Helmi FARID, Montréal, Les éditions Agence d'Arc inc., 1988, 160 pages.

Disons d'emblée de cet ouvrage qu'il s'adresse aux parents d'adolescents. II ne s'agit donc pas d'un livre spécialisé, mais plutôt d'un ouvrage que l'auteur a voulu rendre accessible à tous.

Ce livre, divisé en deux parties, traite dans un premier temps de différents thèmes propres à l'adolescence. Ainsi sont abordées les questions suivantes : corps et sexualité, groupe et amitié, école, autorité parentale, attitudes face aux mass média et à la société actuelle, tabac, alcool et drogue, suicide et, finalement, fugue. Chacune de ces questions fait l'objet d'un chapitre auquel l'auteur a annexé les résultats d'une enquête qu'il a menée auprès de 1932 élèves du secondaire. 